\title{
Resolving the Sub-Rouse Modes by Creep Compliance Measurements in Poly(methyl-para-tolyl-siloxane)
}

\author{
D. J. Plazek ${ }^{1}$ K. L. Ngai, ${ }^{2 *}$
}

${ }^{1}$ Department of Mechanical Engineering and Material Science, University of Pittsburgh, Pittsburgh, Pennsylvania 15261United States

${ }^{2}$ CNR-IPCF, Largo B. Pontecorvo 3, I-56127, Pisa, Italy

*Corresponding author: K. L. Ngai (kiangai@yahoo.com)

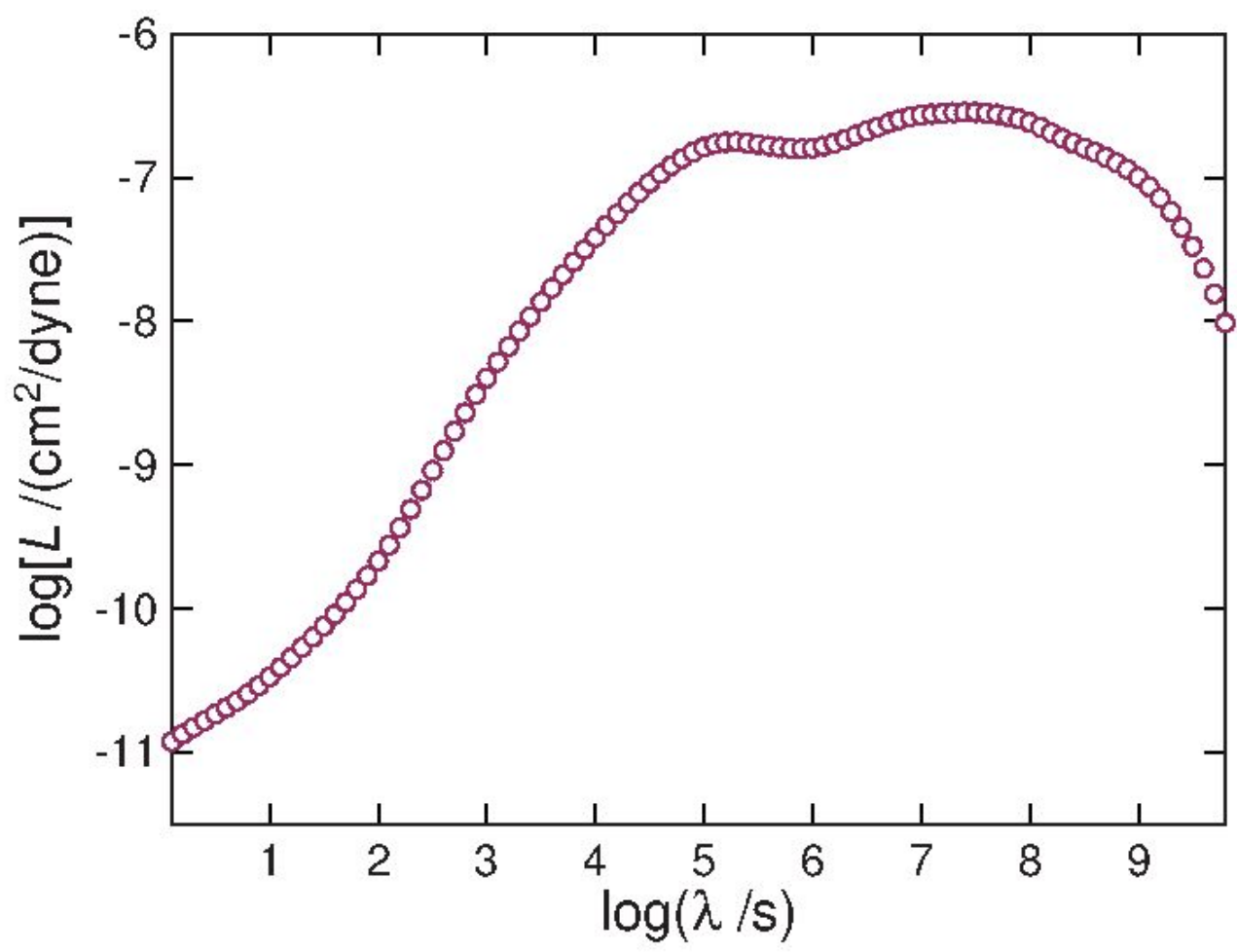

Figure S1. Logarithmic presentation of the retardation spectrum $L$ obtained from the reduced curve of Figure 2 in the paper as a function of the reduced retardation time $\lambda$. 


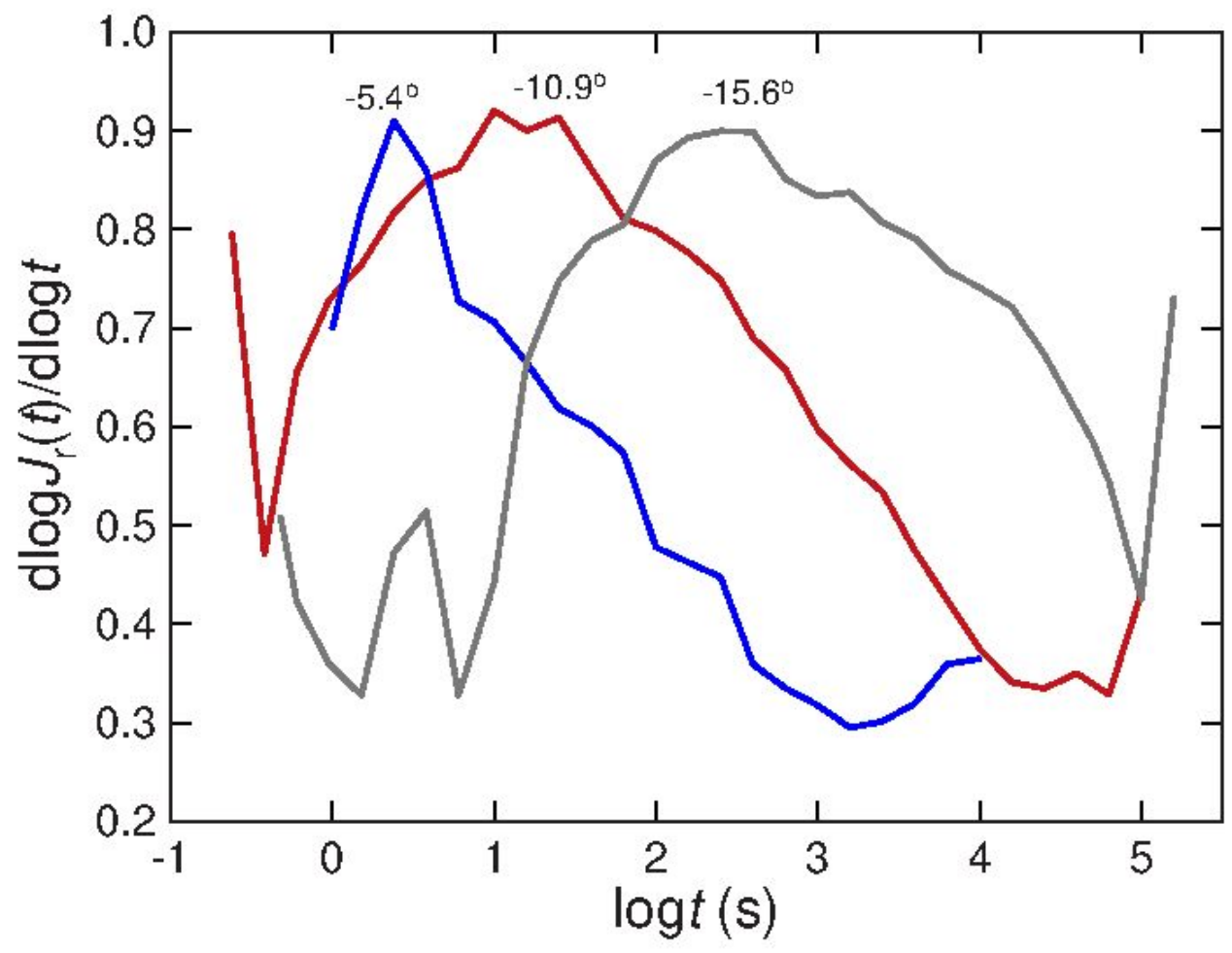

Figure S2. Derivatives obtained from the $J_{r}(t)$ curves in Figure 1 of the paper. 


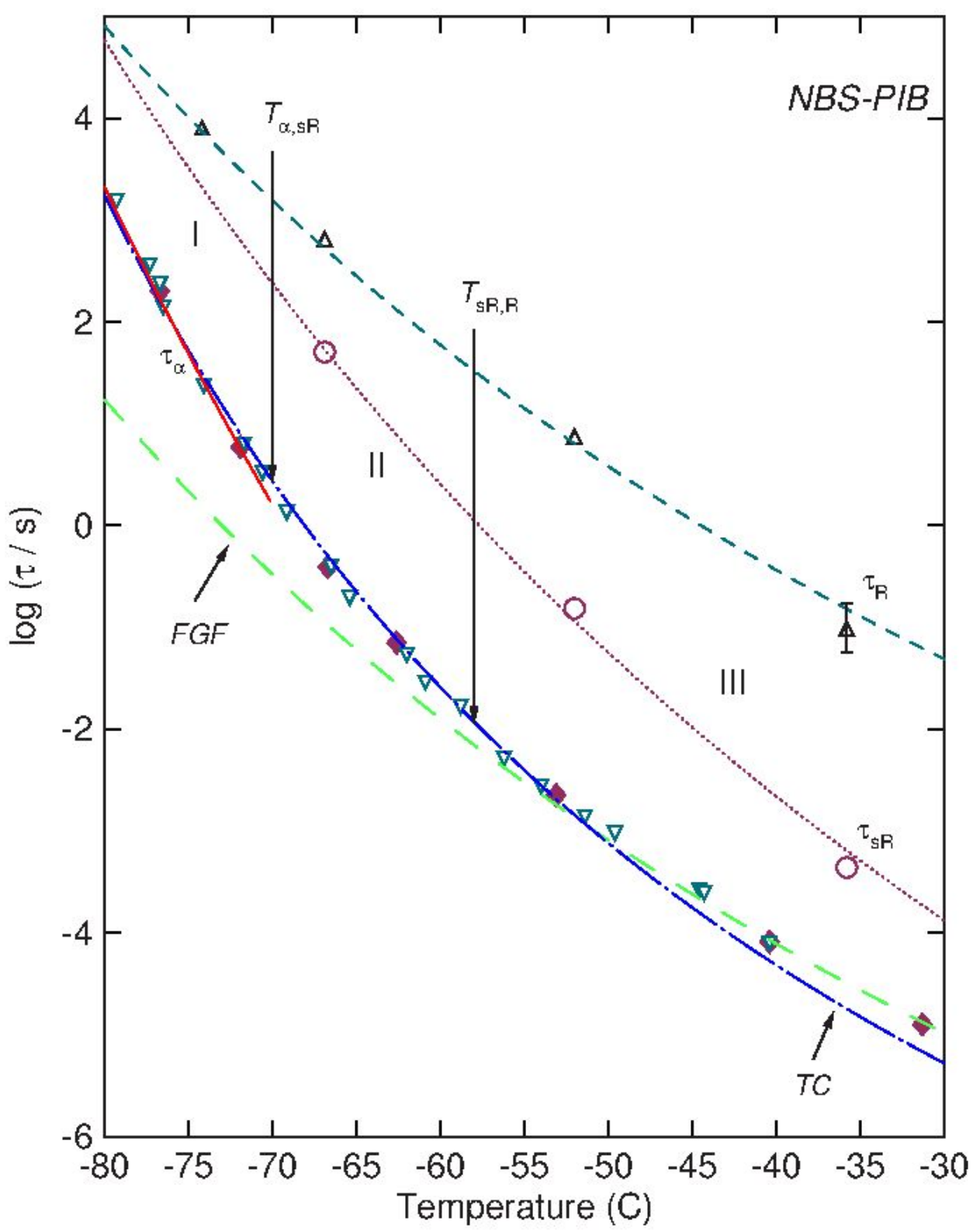

Figure S3. The Rouse time $\tau_{R}$ (open triangles), the sub-Rouse time $\tau_{s R}$ (open circles), and the segmental $\alpha$-relaxation time $\tau_{\alpha}$ (diamonds and inverted triangles) of high molecular weight PIB. The figure is a replot of Fig.1 in ref.S1. 


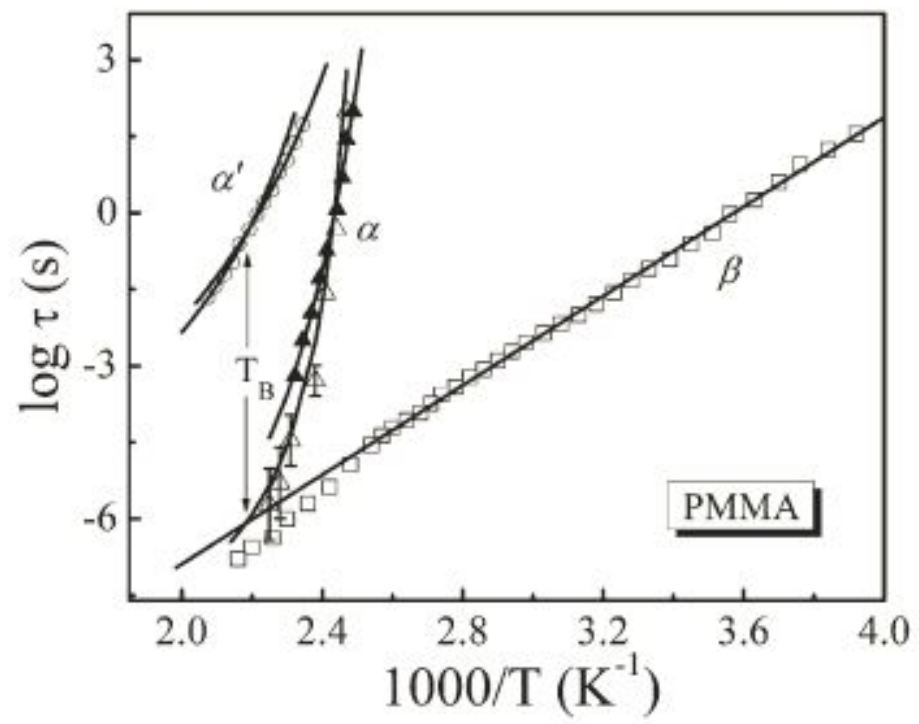

Figure S4. Temperature dependences of characteristic relaxation times of PMMA for mechanical $\alpha(\boldsymbol{\Delta})$ and $\alpha^{\prime}(\mathrm{O})$ relaxations, and dielectric $\alpha(\Delta)$ and $\beta(\square)$ relaxations. The $\beta$ relaxation are fitted by Arrhenius law, and the $\alpha$ relaxations are fitted by a single VFT equation, while the $\alpha^{\prime}$ relaxation is fitted by two VFT equations. Figure reproduced from ref.S2. 


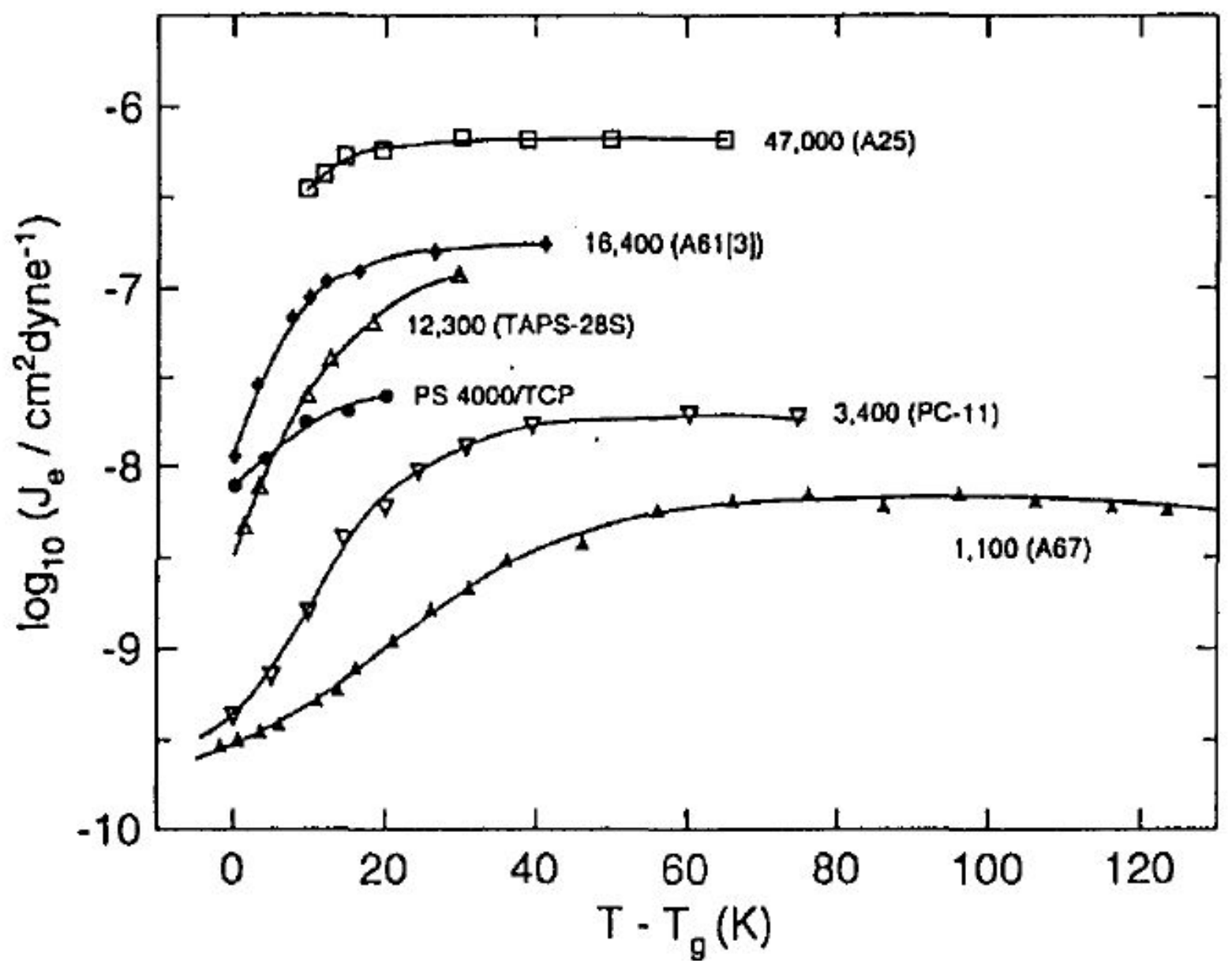

Figure S5 Logarithm of the steady state recoverable compliance, $J_{e}$ plotted against $T-T_{g}$ for several polystyrenes with different molecular weights. Figure reproduced from ref.S3 by permission. 


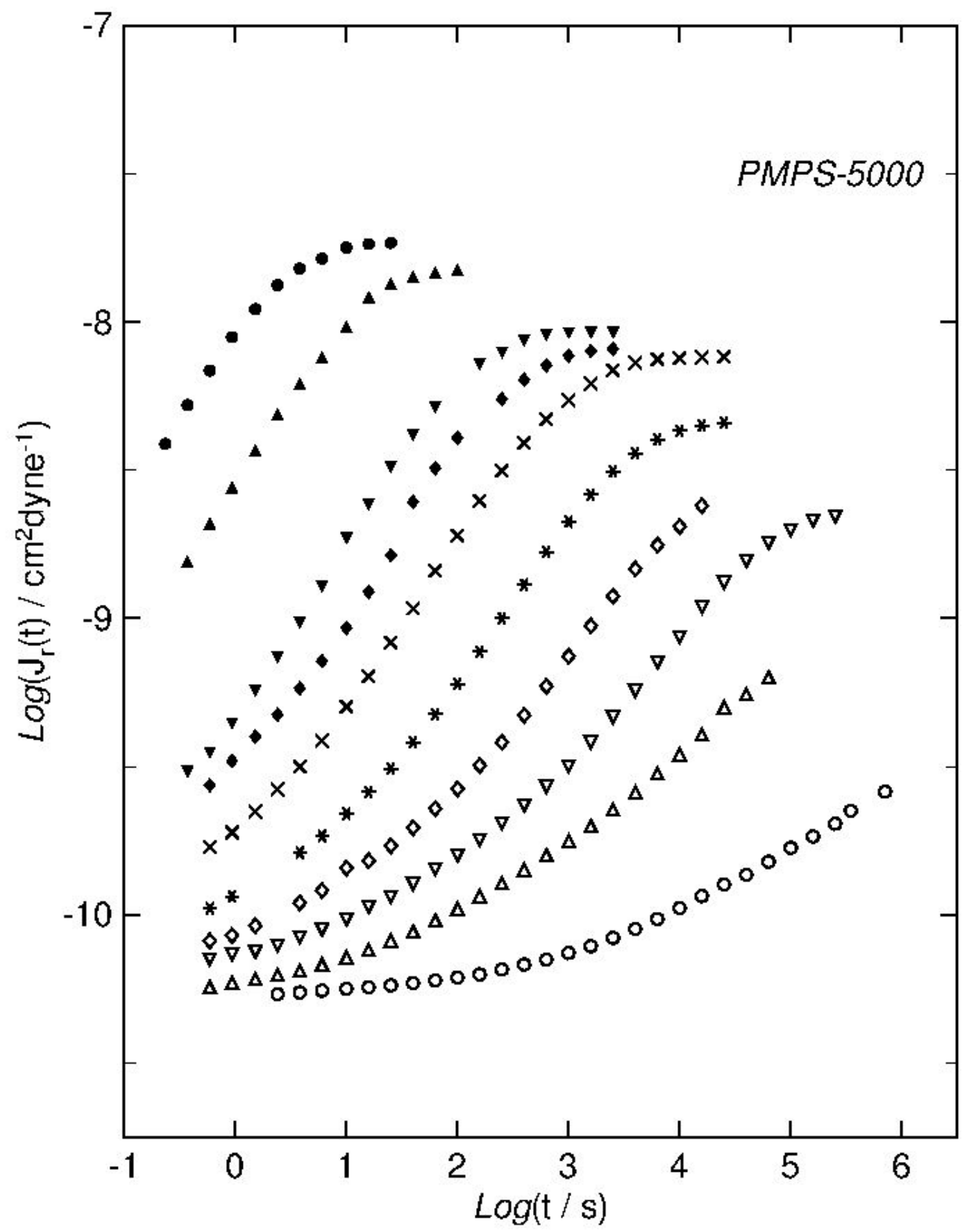

Figure S6 Recoverable compliance, $\mathrm{J}_{\mathrm{r}}(\mathrm{t})$, data of PPMS 5000 at temperatures (from top to bottom): $-32.2^{\circ} \mathrm{C} ;-35.0^{\circ} \mathrm{C} ;-38.6^{\circ} \mathrm{C} ;-40.0^{\circ} \mathrm{C} ;-41.1^{\circ} \mathrm{C} ;-42.6^{\circ} \mathrm{C} ;-44.5^{\circ} \mathrm{C} ;-45.2^{\circ} \mathrm{C} ;-46.9^{\circ} \mathrm{C} ;-50^{\circ} \mathrm{C}$. Figure reproduced from ref.S3 by permission. 


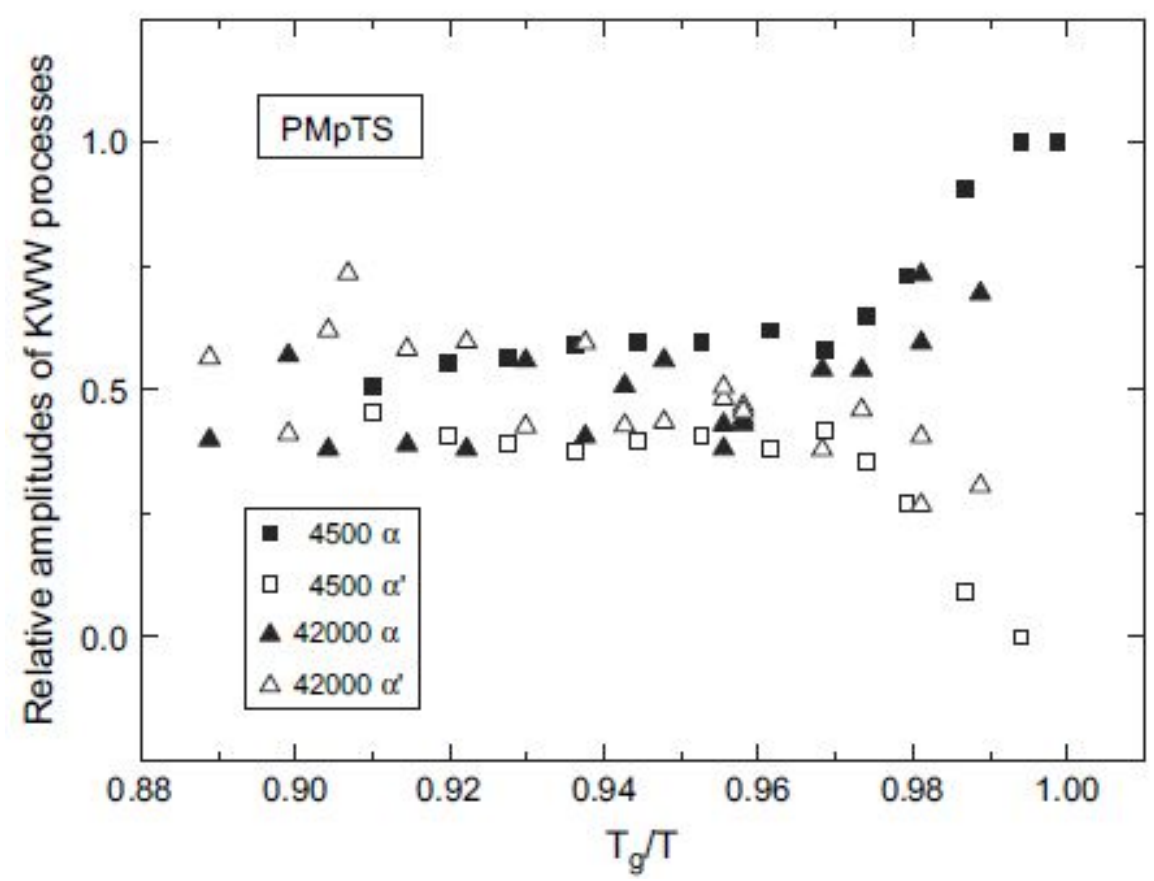

Figure S7 Temperature dependence of the relative amplitudes $A_{\alpha}$ and $A_{\alpha^{\prime}}$ of the $\alpha$ and $\alpha^{\prime}$ processes obtained by fitting the field correlation function $g^{(1)}(t)$ of PCS by eq.(5) in the paper for two PMpTS samples with molecular weights indicated. Reproduced from ref.S5 by permission.

\section{REFERENCES}

(S1) Ngai, K. L.; Plazek, D. J.; Rizos, A. K. Viscoelastic Properties of Amorphous Polymers. 5. A Coupling Model Analysis of the Thermorheological Complexity of Polyisobutylene in the GlassRubber Softening Dispersion, J. Polym. Sci., Part B: Polym.Phys. 1997, 35, 599.

(S2) Wu, X.; Liu, C.; Zhu, Z.; Ngai, K. L.; Wang, L. Nature of the Sub-Rouse Modes in the GlassRubber Transition Zone of Amorphous Polymers. Macromolecules 2011, 44, 3605-3610.

(S3) Ngai, K.L.; Plazek, D.J. IDENTIFICATION OF DIFFERENT MODES OF MOLECULAR MOTION IN POLYMERS THAT CAUSE THERMORHEOLOGICAL COMPLEXITY, Rubb. Chem. Tech. 1995, 68, 376-434.

(S4) D. J. Plazek, C. A. Bero, S. Neumeister, G. Floudas, G. Fytas, and K. L. Ngai, Viscoelastic properties of amorphous polymers 3: low molecular weight poly(methylphenylsiloxane), Colloid Polym Sci 272:1430-1438 (1994).

(S5) Patkowski, A.; Gapinski, J.; Pakula, T.; Meier, G. Physical Nature of Complex Structural Relaxation in Polysiloxane-PMpTS: $\alpha$ and $\alpha^{\prime}$ Relaxations. Polymer 2006, 47, 7231-7240. 